\title{
Serum lipid Profile and Nutritional Status of the Hypertensive Outpatients in University College Hospital, Ibadan
}

\section{Olaitan Oluwasiji Olabisi ${ }^{1 *}$, Fadupin Grace Tanimowo ${ }^{2}$ and} Adebiyi Adewole $\mathbf{A}^{3}$

${ }^{1}$ Nutrition and Dietetic unit, Department of Food Science \& Technology, College of Agriculture, Food science and Technology, Wesley University, Nigeria

${ }^{2}$ Department of Human Nutrition, Faculty of Public Health, University of Ibadan, Nigeria

${ }^{3}$ Cardiology unit, Department of Medicine, University College Hospital, Nigeria

*Corresponding author: Olaitan Oluwasiji Olabisi, Nutrition and Dietetic unit, Department of Food Science \& Technology, College of Agriculture, Food science and Technology, Wesley University, Ondo State, Nigeria, Tel: +2347068518707/ +2348050660012; Email: Sijola2k3@gmail.com

\section{Abstract}

Background: Dyslipidaemia among hypertensive patients increases risks of cardiovascular morbidity and mortality. Nutritional status of the patients relates to the clinical diagnosis in confirming the cause of their ailment. This study aims to evaluate the lipid profile and its relationship with nutritional status of hypertensive outpatients attending University College Hospital in Ibadan.

Methodology: This was a descriptive cross-sectional study involving the fifty-five (55) alternative hypertensive outpatients(32 females and 23 males) attending cardiology clinics of Medical Outpatients in University College Hospital, Ibadan, Oyo State, Nigeria. A semi-structured, interviewer-administered questionnaire was used to obtain patients' socio-economic data. The mean of the last four (4) blood pressure readings and lipid profile of the patients were obtained from their hospital records. Anthropometric parameters, weight (kg), height (m), waist and hip circumferences of the patients were assessed. Descriptive and inferential statistics were employed in data analysis. Level of significance was placed at $\mathrm{p}<0.05$.

Results: The mean age of the patients was $56.7 \pm 12.3$. Less than half (45.5\%) of the patients were between the age 50 64years. The mean BMI of the patients was $28.7 \pm 26.12\left(\mathrm{~kg} / \mathrm{m}^{2}\right)$. A large number $(36.4 \%)$ of patients were found to be overweight, (34.5\%) were obese and (29.1\%) had normal weight. There were more (46.9\%) female patients obese than male patients. Large number (73.9\%) male and (84.4\%) female patients had waist-hip ratio above normal cutoff points for both sexes. Male patients had higher mean low lipoprotein, high density lipoprotein and triglycerides than female counterparts while female patients had higher mean total cholesterol than their male counterparts. A significant difference was observed in total cholesterol and low density lipoprotein profile between the male and 


\section{International Journal of Biochemistry \& Physiology}

female patients $(\mathrm{p}<0.05)$. A positive correlation between hypertension and waist-hip ratio, and serum total cholesterol was also observed only among the male patients.

Conclusion: Many of the patients were overweight and obese. Most of them suffered from diabetes mellitus which they were not aware of. Large numbers of the patients lived a sedentary life. There was raised serum total cholesterol, and decreased high density lipoprotein especially among the patients.

Keywords: Lipid profile; Nutrition status; Hypertension; Outpatients

Abbreviations: VLDL-C: Very-Low-Density Lipoprotein Cholesterol; HDL-C: High-Density Lipoprotein Cholesterol; TG: Triglycerides; TC: Total Cholesterol; SPSS: Statistical Package for Social Science; NCEP: American National Cholesterol Education Program; HDL: High Density Lipoprotein; LDL: Low Density Lipoprotein.

\section{Introduction}

Hypertension is one of the chronic noncommunicable diseases which pose public health challenge in developing countries especially among the black race where it is most prevalent [1]. It is the number one cardiovascular disease which affects approximately 1 billion people globally and it accounts for about 7.5 million deaths annually, which is about $12.8 \%$ of all total deaths [2]. It is confirmed when blood pressure is sustained at $\geq 140 / 90 \mathrm{mmHgafter}$ two or more systolic and diastolic blood pressure readings [3], and dyslipidemia is one of its risk factors $[4,5]$.

Dyslipidemia, also referred to as hyperlipidemia, is an abnormal serum lipid which is characterized by elevated plasma total cholesterol (TC), triglycerides (TG), and TG-rich very-low-density lipoprotein cholesterol (VLDL-C), reduced high-density lipoprotein cholesterol (HDL-C). It contributes significantly to excess risk of cardiovascular diseases among which is hypertension [6].

The relationship between hypertension and several lipid abnormalities involves elevated total cholesterol (TC) concentrations and the presence of dyslipidemia increases risks of cardiovascular morbidity and mortality [4].

The predisposing factors of the hyperlipidemia identified include increased consumption of dietary animal fat, lack of physical exercise, genetic factors, severe stress, increased age, sex, alcohol and tobacco consumption, and metabolic disorders like diabetes mellitus and hypothyroidism. Serum lipid level of hypertensive patients which is usually higher, can however be lowered by dietary restriction and hypolipidemic agents $[4,7]$.

Excessive daily intake of saturated fats, cholesterol, other sources of calorie and subsequent lipid profile have been established to contribute to hypertriglyceridemia among the obese-hypertensive individuals, and hypercholestemia resulting to obesity and consequently hypertension [8]. Furthermore, hyperlipidemia also substantially worsens the prognosis in hypertensive patients [9].

This therefore necessitates a need to examine the serum lipid profile and nutritional status of the hypertensive patients in order to have holistic clinical examination in diagnosis of hypertension. The aim of this study is to examine the serum lipid profile and nutritional status of the hypertensive patients attending tertiary hospitals especially medical outpatient clinics in the University College Hospital, Ibadan in Oyo state, Nigeria in order to determine the contribution of the patients' serum lipid profile and nutritional status to the episode of the hypertension status so that information gathered could be useful in the management of hypertension among the hypertensive patients in the hospitals.

\section{Methodology}

\section{Study Design}

The study was a descriptive cross-sectional in design to assess the association between serum lipid profile and nutritional status of hypertensive patients.

\section{Study Location}

Study was carried out at the University College hospital, Ibadan Oyo State, Nigeria. A total of fifty-five (55) hypertensive outpatients (32 females and 23 males) attending Cardiology Clinics of Medical Outpatients (MOP) of the University College Hospital, Ibadan, Oyo State, Nigeria. 


\section{International Journal of Biochemistry \& Physiology}

\section{Data Collection Technique}

A semi-structured, interviewer-administered questionnaire was used to interview each patient that gave their consent to participate in the study. The patients' hospital records were used to obtain the last four (4) blood pressure levels and lipid profile. Weight (kg), Height (m), Percentage body fat, visceral fat and resting metabolism of the respondents were measured using body composition device (Omron instrument).Waist and Hip circumferences (cm) of the respondents was assessed by using measuring tape.

Information on the patients' socio-demographic data namely; age, sex, tribe, religion, income, occupation and educational status were obtained with the aid of the semi-structured questionnaire. The nutrition status of the patients was assessed by measuring their weight, height, waist and hip circumferences. The Patients' Body Mass Index and waist-to-hip ratio were calculated for the two sexes. BMI was classified into underweight, normal weight, overweight and obesity. The classification of waist-to-hip ratio was based on the specification of World Health Organisation [1].

According to the Third Report of the National Cholesterol EducationProgram on Detection, Evaluation, and Treatment of High Blood Cholesterol in Adults (Adult Treatment Panel III) for the serum lipid profile reference level, hypercholesterolemia was defined as TC $>200 \mathrm{mg} / \mathrm{dl}$, high LDL-C when value is $\geq 130 \mathrm{mg} / \mathrm{dl}$, hypertriglyceride as TG $>150 \mathrm{mg} / \mathrm{dl}$, and low HDL-C as $<40 \mathrm{mg} /$ dl [10]. Dyslipidemia was defined by presence of one or more abnormal lipid concentration. The target for normal lipid values are LDL $<130 \mathrm{mg} / \mathrm{dl}$, HDL $>40 \mathrm{mg} / \mathrm{dl}$, Triglyceride $<150 \mathrm{mg} / \mathrm{dl}$ and Total cholesterol $<200 \mathrm{mg} / \mathrm{dl}$.

\section{Data Analysis}

The data collected were evaluated by using Statistical Package for Social Science (SPSS) 20.0 version software. Descriptive statistics was measured for mean, standard deviation, frequency and percentage. Tabulation of the variables was done. T-test and ANOVA were used to compare betweenthe mean differences across the gender. Pearson's correlation was performed to determine association between hypertension and lipid profile. The result was considered significant when $\mathrm{p} \leq 0.05$.

\section{Results}

A total of 55 patients were interviewed; $23(41.8 \%)$ males and $32(58.2 \%)$ females were recruited for the study. The mean age of the patients was $56.7 \pm 12.3$. Less than half $(45.5 \%)$ of the patients were between the age 50-64years. Majority of the patients were Yoruba $(83.6 \%)$ while others were Igbo, Edo, Iroboand Isoko. Most $(89.1 \%)$ of the patients were married and $80.0 \%$ were monogamous. A large number $(80.0 \%)$ had either secondary or tertiary education.

\section{Serum Lipid Profile and Blood Pressure of the Hypertensive Patients}

The result presented in table 1 shows the analysis of variance of lipid profile of male and female hypertensive patients. The mean total cholesterol levels of male and female patients were $177.4 \pm 29.44 \mathrm{mg} / \mathrm{dl}$ and $197.5 \pm 38.52 \mathrm{mg} / \mathrm{dl}$ respectively. Average low density lipoproteins of male and female patients were $104.5 \pm 31.99 \mathrm{mg} / \mathrm{dl}$ and $127.9 \pm 31.71 \mathrm{mg} / \mathrm{dl}$ respectively. Average high density lipoproteins of male and female patients were $51.2 \pm 19.89 \mathrm{mg} / \mathrm{dl}$ and $47.1 \pm 10.18 \mathrm{mg} / \mathrm{dl}$ respectively. Mean triglycerides of male and female patients were $107.1 \pm 87.60 \mathrm{mg} / \mathrm{dl}$ and $117.7 \pm 43.66 \mathrm{mg} / \mathrm{dl}$ respectively.

Average Systolic blood pressure readings of the male and female patients were $148 \pm 15.3 \mathrm{mmHg}$ and $143 \pm 15.4 \mathrm{mmHg}$ while diastolic blood pressure readings of the male and female patients were $90 \pm 8.9 \mathrm{mmHg}$ and $85 \pm 10.7 \mathrm{mmHg}$. A significant difference was observed in total cholesterol and low density lipoprotein profile between the male and female patients $(\mathrm{p}<0.05)$.

\section{Nutritional Status of the Patients}

The mean Body Mass Index of the patients was $28.7 \pm 26.12\left(\mathrm{~kg} / \mathrm{m}^{2}\right)$ (Table 2). According to result presented on Table 3, a significant difference was observed in BMI between the male and female patients. A large number of the patients were found to be overweight and obese (65.5\%) combined while only few $(29.1 \%)$ of the patients had normal weight. There were more $(46.9 \%)$ female patients obese than male patients (Table 2).

The result (Figure 1) of the patients' waist and hip circumferences shows that large number (73.9\%) male and (84.4\%) female patients had waist-hip ratio above normal cut-off points of 0.90 and 0.85 for male and female respectively. This expresses that most of the patients were at the risk of metabolic disorders (such as hypertension, obesity, diabetes mellitus and others). The male patients were found to have higher visceral fat, muscle, resting metabolism, and to be taller and heavier than women. However, female patients had more body fat than their male counterparts. A significant mean difference was observed in height, BMI, body fat, muscle and resting metabolism between male and female patients (Table 3). 


\section{International Journal of Biochemistry \& Physiology}

\section{Correlation between Lipid Profile and Hypertension of the Patients}

There is a significant positive correlation between hypertension and total cholesterol and high density lipoprotein only among the male patients (Table 4).

\section{Body Mass Index and Lipid Profile of the Hypertensive Patients}

According to the result presented on the table 5, no association was found between the serum lipid profile and nutritional status of the hypertensive patients.

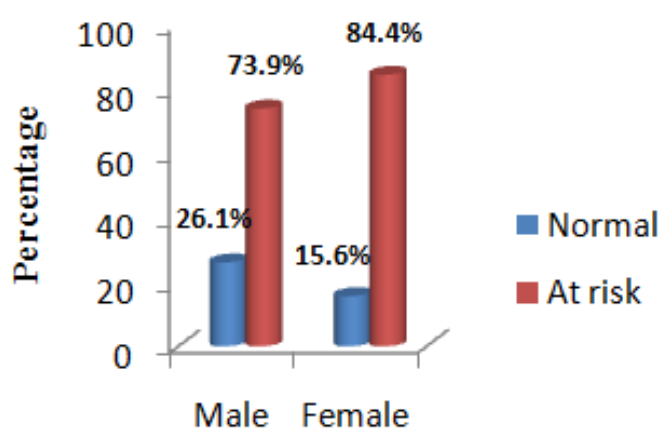

Figure 1: Patients' waist-to-hip ratio

\begin{tabular}{|c|c|c|c|c|c|c|}
\hline Variables & \multicolumn{2}{|c|}{ Male } & \multicolumn{2}{c|}{ Female } & F & P-value \\
\hline & x(SD) Range & Range & $\mathbf{x ( S D )}$ & Range & & \\
\hline $\mathrm{TC}(\mathrm{mg} / \mathrm{dl})$ & $177(29.4)$ & $199-227$ & $197.5(38.5)$ & $135-303$ & 4.375 & $0.041^{*}$ \\
\hline $\mathrm{LDL}(\mathrm{mg} / \mathrm{dl})$ & $105(31.9)$ & $51-145$ & $127.9(31.7)$ & $80-204$ & 5.171 & $0.027^{*}$ \\
\hline $\mathrm{HDL}(\mathrm{mg} / \mathrm{dl})$ & $51(19.89)$ & $19-93$ & $47.1(10.2)$ & $26-76$ & 0.977 & 0.327 \\
\hline $\mathrm{TG}(\mathrm{mg} / \mathrm{dl})$ & $107(87.6)$ & $53-486$ & $117.7(43.7)$ & $55-294$ & 0.347 & 0.558 \\
\hline $\mathrm{SBP}(\mathrm{mmHg})$ & $148(15.3)$ & $115-175$ & $143(15.4)$ & $110-177$ & 1.354 & 0.25 \\
\hline $\mathrm{DBP}(\mathrm{mmHg})$ & $90(8.9)$ & $76-105$ & $85(10.7)$ & $65-117$ & 2.957 & 0.091 \\
\hline
\end{tabular}

Table 1: Summary of Analysis of Variance of Lipid Profile and Blood Pressure of Patients.

TC- Total Cholesterol, LDL-Low Density Lipoprotein, HDL-High Density Lipoprotein, TG- Triglyceride, SBP- Systolic Blood Pressure, DBP- Diastolic Blood Pressure, x-Mean, SD- Standard deviation. *Significant at $\mathrm{p}<0.05$.

\begin{tabular}{|c|c|c|c|c|c|}
\hline BMI $\left(\mathrm{Kg} / \mathrm{m}^{2}\right)$ & $\begin{array}{c}\text { Male } \\
\text { N(\%) }\end{array}$ & $\begin{array}{c}\text { Female } \\
\mathrm{N}(\%)\end{array}$ & Total & $\mathrm{X}^{2}$ & p-value \\
\hline$<18.5$ & $0(0.0)$ & $0(0.0)$ & $0(0.0)$ & \multirow{5}{*}{5.287} & \multirow{5}{*}{0.071} \\
\hline $18.5-24.9$ & $9(39.1)$ & $7(21.8)$ & $16(29.1)$ & & \\
\hline $25.0-29.9$ & $10(43.5)$ & $10(31.3)$ & $20(36.4)$ & & \\
\hline$\geq 30.0$ & $4(17.4)$ & $15(46.9)$ & $19(34.5)$ & & \\
\hline Total & $23(41.8)$ & $32(58.2)$ & $55(100.0)$ & & \\
\hline
\end{tabular}

Table 2: Body Mass Index of the Hypertensive Patients.

\begin{tabular}{|c|c|c|c|c|c|c|}
\hline \multirow{2}{*}{ Variables } & \multicolumn{2}{|c|}{ Male } & \multicolumn{2}{|c|}{ Female } & \multirow{2}{*}{$\mathbf{F}$} & \multirow{2}{*}{ P-value } \\
\hline & $\mathrm{n}(\mathrm{SD})$ & Range & $\mathrm{n}(\mathrm{SD})$ & Range & & \\
\hline BMI $\left(\mathrm{kg} / \mathrm{m}^{2}\right)$ & 26.1(3.84) & $18.60-34.50$ & $28.7(4.51)$ & $20.50-35.80$ & 4.964 & $0.030^{*}$ \\
\hline WC (m) & $0.9(0.92)$ & $0.78-1.11$ & $0.9(0.16)$ & $0.42-1.73$ & 0.201 & 0.656 \\
\hline $\mathrm{HC}(\mathrm{m})$ & $1.0(0.14)$ & $0.48-1.14$ & $1.0(0.15)$ & $0.43-1.34$ & 2.618 & 0.112 \\
\hline Waist/Hip ratio & $0.9(0.35)$ & $0.05-2.31$ & $0.9(0.09)$ & $0.75-1.15$ & 0.24 & 0.626 \\
\hline Body Fat (\%) & $25.1(7.00)$ & $9.50-39.50$ & $39.2(7.54)$ & $19.70-50.20$ & 50.064 & $0.000^{*}$ \\
\hline Visceral Fat (\%) & $10.9(4.74)$ & $2.00-22.00$ & 9.4(3.28) & $2.00-18.00$ & 1.866 & 0.178 \\
\hline Muscle (\%) & $32.8(3.89)$ & $25.20-41.10$ & $26.5(5.63)$ & $21.30-51.70$ & 21.677 & $0.000^{*}$ \\
\hline $\mathrm{RM}($ Kcal $)$ & $1615(165.83)$ & $1141-1878$ & $1462(149.06)$ & $1225-1835$ & 12.824 & $0.001^{*}$ \\
\hline
\end{tabular}

Tables 3: Analysis of Variance of the Anthropometric/Body Composition and Resting Metabolic Indices of the Patients.

BMI- Body Mass Index, WC- Waist circumference, HC- Hip circumference, RM- Resting Metabolism,n-Mean, SDStandard deviation. *Significant at $\mathrm{p}<0.05$. 


\section{International Journal of Biochemistry \& Physiology}

\begin{tabular}{|c|c|c|c|c|c|c|c|c|c|c|}
\hline \multirow{2}{*}{ Variables } & \multicolumn{2}{|c|}{ Total Cholesterol } & \multicolumn{2}{|c|}{ LDL } & \multicolumn{2}{c|}{ HDL } & \multicolumn{2}{c|}{ TRG } & \multicolumn{2}{c|}{ Waist-hip ratio } \\
\cline { 2 - 11 } & $\mathbf{R}$ & P value & R & P value & R & P value & R & P value & R & P value \\
\hline Male & 0.413 & $0.050^{*}$ & 0.278 & 0.199 & 0.414 & $0.050^{*}$ & 0.043 & 0.846 & 0.544 & $0.007^{*}$ \\
\hline Female & 0.031 & 0.868 & 0.027 & 0.883 & -0.187 & 0.305 & 0.173 & 0.343 & 0.384 & $0.030^{*}$ \\
\hline
\end{tabular}

Table 4: Correlation between Hypertension, Lipid Profile and Waist-hip ratio of the Patients.

\begin{tabular}{|c|c|c|c|c|c|c|c|c|}
\hline \multicolumn{9}{|c|}{ Lipid Profile } \\
\hline \multirow{2}{*}{ BMI $\left(\mathrm{Kg} / \mathrm{m}^{2}\right)$} & \multicolumn{2}{|c|}{ TC } & \multicolumn{2}{|c|}{ TG } & \multicolumn{2}{|c|}{ HDL } & \multicolumn{2}{|c|}{ LDL } \\
\hline & Normal & At risk & Normal & At risk & Normal & At risk & Normal & At risk \\
\hline$<18.5$ & $0(0.0)$ & $1(100.0)$ & $1(100.0)$ & $0(0.0)$ & $1(100.0)$ & $0(0.0)$ & $1(100.0)$ & $0(0.0)$ \\
\hline $18.5-24.9$ & $13(86.7)$ & $2(13.3)$ & $15(100.0)$ & $0(0.0)$ & $12(80.0)$ & $3(20.0)$ & $7(46.7)$ & $8(53.3)$ \\
\hline $25.0-29.9$ & $12(60.0)$ & $8(40.0)$ & $18(90.0)$ & $2(10.0)$ & $18(90.0)$ & $2(10.0)$ & $16(80.0)$ & $4(20.0)$ \\
\hline$\geq 30.0$ & $10(52.9)$ & $9(47.1)$ & $16(84.2)$ & $3(15.8)$ & $16(84.2)$ & $3(15.8)$ & 14(73.7) & $5(26.3)$ \\
\hline Total & $35(63.6)$ & $20(36.4)$ & $50(90.9)$ & $5(9.1)$ & $47(85.5)$ & $8(14.5)$ & $38(69.1)$ & $17(30.9)$ \\
\hline p-value & \multicolumn{2}{|c|}{0.098} & \multicolumn{2}{|c|}{0.829} & \multicolumn{2}{|c|}{0.152} & \multicolumn{2}{|c|}{0.449} \\
\hline $\mathrm{X}^{2}$ & \multicolumn{2}{|c|}{6.297} & \multicolumn{2}{|c|}{0.885} & \multicolumn{2}{|c|}{5.282} & \multicolumn{2}{|c|}{2.652} \\
\hline
\end{tabular}

Table 5: Association between Body Mass Index and Lipid Profile of the Hypertensive Patients.

\section{Discussion}

This study shows that hypertension is prevalent among the patients within the age of 50-64years. This confirms the reports of many studies within and outside Nigeria which establish the association between age and hypertension $[11,12]$. Elisabete indicated that increase in hypertension in the older adult was due to the structural changes in the arteries especially large artery and stiffness associated with ageing [12].

Having majority of the respondents in this study to be Yoruba is attributed to the research location. The remaining patients come from other ethnics which include; Igbo, Isoko and Edo. It was also observed that hypertension was more prevalent among the females than their male counterparts. This disparity supports the claim of Megan, et al. (2008) that menopause contributes to high blood pressure among the older women [5].

On the average, this study observed that female patients had raised blood cholesterol against the cut off points given by the American National Cholesterol Education Program (NCEP) Expert Panel for hypertension and other Coronary Heart Diseases (2003) more than male patients. Not that alone, the female patients were also found to have more body fat than their male counterparts. This is traceable to postmenopausal effect in which older women have their estrogen reduced and body fat increased [13].

Many previous studies had observed negative correlation between blood lipid and elevated blood pressure among the hypertensive patients $[7,14,15]$.
However the result of this study shows that the serum lipid profile (including total cholesterol and high density lipoprotein) is found to be positively correlated to hypertension most especially among male hypertensive patients. Low density lipoprotein (LDL) and triglyceride (TG) have no significant changes with hypertension for both sexes. The association of hypertension with raised total cholesterol (TC) and lowered high density lipoprotein (HDL) among the hypertensive patients in this research is established to be the result of overweight and obesity which are rampant among the patients.

Some studies done inside and outside Nigeria had established raised total cholesterol among the hypertensive patients $[4,7]$. This study also confirmed higher serum cholesterol among the hypertensive patients with significant correlation between hypertension and total cholesterol observed only among male patients.

In contrast, the study of Onwubere, et al., (2011) reported that no correlation between hypertension and total cholesterol among the hypertensive patients in their study [15]. The increased in serum total cholesterol among the hypertensive patients in our study is due to the genetic factor, and other factors such as; increased consumption of dietary animal fats, lack of physical exercise, claimed stress, and old age which were found to be true among the hypertensive patients. This finding is supported by the report of Akuyam, et al. (2009) in their study conducted among the hypertensive and normotensive individuals in Zaria, Nigeria [4]. 


\section{International Journal of Biochemistry \& Physiology}

The result of this study on low density lipoprotein and Triglycerides is contrary to the findings of Saha, et al. who reported higher level of low density lipoprotein and Triglycerides among the hypertensive patients [7]. However, the significant higher low density lipoprotein noticed among the male patients is due to the metabolic disorders such diabetes mellitus which result from the excessive weight gain among them.

The Body Mass Index of the patients revealed that most of the patients were overweight and obese. Women were found to be more obese than men while men were more overweight than women. This explains the reason why almost all the women and greater number of men in this study had higher waist-hip ratio than recommended. Sedentary lifestyle of the hypertensive patients also influences the excessive weight gain, and lack of job among the elderly makes most of them to stay at home and physically inactive $[16,17]$.

Most of the patients under this study had waist -tohip ratio higher than normal cut off points for healthy life as recommended by World Health Organisation for both adult male and female [1]. The waist to hip ratio of the patients was found significantly associated with hypertension $(p<0.05)$. This suggests that many of the patients were suffering from metabolic disorders such as diabetes and obesity which are precursors to cardiovascular diseases. This similar case of obesity among the hypertensive patients was reported by Adediran, et al. in Abuja where obesity was found to be more prevalent among urban dwellers who were hypertensive than rural dwellers who could engage in physical activity with their farming work [11]. Yuko, et al. also confirmed prevalence of obesity among hypertensive outpatients in Fukuoka, Japan [2].

\section{Conclusion}

It is established from this study that hypertension is prevalent among the young adults and elderly patients attending University College Hospital, Ibadan. There is raised serum total cholesterol; and decreased high density lipoprotein among the female patients. A lot of the patients were overweight and obese. Most of the patients were suffering from diabetes mellitus which they might not be aware of. A significant correlation was found between total cholesterol, waist-hip ratio and hypertension among the hypertensive patients.

\section{Recommendation}

The hypertensive patients should be encouraged to engage in physical activities and receive counseling on how to maintain normal weight as a means to prevent the risk of obesity and diabetes which can complicate their condition. The patients need to also undergo test to check their blood sugar level so as to know their medical status for diabetes.

\section{References}

1. World Health Organisation (2008) Waist Circumference and Waist-Hip Ratio: Report of a WHO Expert Consultation, Geneva.

2. Ohta Y, Tsuchihashi T, Kiyohara K (2011) Relationship between Blood Pressure Control Status and Lifestyle in Hypertensive Outpatients. An Article of Internal Medicine 50(19): 2107-2112.

3. Mancia G, Fagard R, Narkiewicz K, Redón J, Zanchetti A (2013) 2013 ESH/ESC Guidelines for the management of arterial hypertension: the Task Force for the management of arterial hypertension of the European Society of Hypertension (ESH) and of the European Society of Cardiology (ESC). J Hypertens 31(7): 1281-1357.

4. Akuyam SA, Aghogho UB, Aliyu IS, Bakari AG (2009) Serum total cholesterol in hypertensive Northern Nigerians. International Journal of Medicine and Medical Sciences 1(3): 073-078.

5. Raksha G, Nandini S (2014) A Correlative Study of Hypertension with Lipid Profile. International Journal of Research in Applied. Natural and social sciences 2(2): 143-150.

6. Manohar SM, Vaikasuvu SR, Deepthi K, Sachan A, Narasimha SR (2013) An association of hyperglycemia with plasma malondialdehyde and atherogenic lipid risk factors in newly diagnosed Type 2 diabetic patients. J Res Med Sci 18(2): 89-93.

7. Saha MS, Sana NK, Ranajit KS (2006) Serum Lipid Profile of Hypertensive Patients in the Northern Region of Bangladesh. J bio-Sci 14: 93-98.

8. Kotsis V, Stabouli S, Papakatsika S, Rizos Z, Parati G (2010) Mechanisms of Obesity-induced Hypertension. Hypertens Res 33(5): 386-393.

9. Harvey JM, Beevers DG (1990) Biochemical Investigation of Hypertension. Ann Clin Biochem 27: 287-296.

10. Chobanian AV, Bakris GL, Black HR, Cushman WC, Green LA, et al. (2003) Seventh Report of the Joint National Committee on Prevention, Detection, Evaluation, and Treatment of High Blood Pressure. Hypertension 42(6): 1206-1252. 


\section{International Journal of Biochemistry \& Physiology}

11. Adediran OS, Okpara IC, Adeniyi OS, Jimoh AK (2013) Hypertension Prevalence in an Urban and Rural Area of Nigeria. Journal of Medicine and Medical Sciences 4(4): 149-154.

12. Elisabete P (2007) Blood pressure and Ageing. Postgrad Med J 83(976): 109-114.

13. Coylewright M, Reckelhoff JF, Ouyang P (2008) Menopause and Hypertension: An Age-Old Debate. Hypertension 51(4): 952-959.

14. Ferrara LA, Guida L, Iannuzzi R, Celentano A, Lionello F (2002) Serum Cholesterol affects Blood Pressure Regulation. J Hum Hypertens 16(5): 337343.

15. Onwubere BJC, Ejim EC, Okafor CI, Emehel A, Mbah $\mathrm{AU}$, et al. (2011) Pattern of Blood Pressure Indices among the Residents of a Rural Community in South East Nigeria. International Journal of Hypertension 621074: 1-6.

16. Yaméogo NV, Kagambèga LJ, Millogo RC, Kologo KJ, Yaméogo AA, et al. (2012) Factors Associated with poor Blood Pressure Control in Hypertensive Black Africans: Cross-sectional Study of 456 hypertensive patients from Burkina Faso. Ann Cardiol Angeiol (Paris) 62(1): 38-42.

17. Zafar SN, Gowani SA, Irani FA, Ishaq M (2008) Awareness of the Risk Factors, presenting features and Complications of Hypertension amongst hypertensive and normotensives. J Pak Med Assoc 58(12): 711-715. 